\title{
An Insight into Oral Leukoplakia
}

\author{
Sham Kishor Kanneppady ${ }^{*}$, Sowmya Sham Kanneppady ${ }^{2}$, Anusha Rangare Lakshman ${ }^{3}$, \\ Shishir Ram Shetty ${ }^{4}$ and Prasanna Kumar $\mathbf{R a o}^{5}$ \\ ${ }^{1}$ Division of Oral Diagnostics and Surgical Sciences, International Medical University, Malaysia \\ ${ }^{2}$ Department of Pharmacology, Faculty of Medicine, Lincoln University College, Malaysia \\ ${ }^{3}$ Department of Oral Medicine and Radiology, Century International Institute of Dental Sciences and Research Centre, India \\ ${ }^{4}$ Department of Oral Medicine and Radiology, Gulf Medical University, UAE \\ ${ }^{5}$ Department of Oral Medicine and Radiology, A.J. Institute of Dental Sciences, India
}

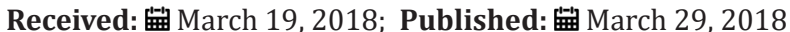

*Corresponding author: Sham Kishor Kanneppady, Senior Lecturer, Division of Oral Diagnostics and Surgical Sciences, School of Dentistry, International Medical University, Kuala Lumpur, Malaysia

\begin{abstract}
Oral leukoplakia (OL) clinically appears as either white or red and white non-scrapable lesion is the most common potentially malignant disorder of the oral mucosa. Diagnosis of OL can be ruled out if an etiologic factor for the whitish plaque can be established, except in the case of smoking. Infections by Candida, human papillomavirus (HPV) and more recently Epstein Barr virus (EBV) have been identified as cofactors that may affect the prognosis of established OL. It is often found among men, and its prevalence increases with age advancement. It has been estimated that it mainly affects men over 40 years. The management of this common condition includes local, topical, and systemic therapies such as anti-oxidants, carotenoids, and antifungal therapies.
\end{abstract}

\section{Introduction}

Oral leukoplakia clinically appears as either white or red and white non scrapable lesion in any portion of oral mucosa, which is associated with tobacco smoking or chewing habit. In 2012 van der Waal proposed a new definition which seems more opportune as it includes the histological confirmation "A predominantly white lesion or plaque of questionable behaviour having excluded, clinically and histopathologically, any other definable white disease or disorder" [1].

\section{Incidence and demographic distribution of oral leuko- plakia}

The estimated prevalence rate of OL worldwide in 2003 varied between 1.7 to $2.7 \%$ in general population. This estimated rate is obtained through a meta-analysis which included 23 primary studies from all over the world published in the period 1986-2002. There was no difference between geographical areas and between younger and older patients. It has been reported that between $16 \%$ and $62 \%$ of OSCC are associated with OL [2]. This disease is often found among men, and its prevalence increases with age advancement. It has been estimated that it mainly affects men over 40 years [3].

\section{Etiology and pathogenesis of oral leukoplakia}

Diagnosis of OL can be ruled out if an etiologic factor for the whitish plaque can be established, except in the case of smoking. Infections by Candida, human papillomavirus (HPV) and more recently Epstein Barr virus (EBV) have been identified as cofactors that may affect the prognosis of established OL [4].

\section{Smoking}

Tobacco smoke is a powerful carcinogen and considered along with chronic alcoholism as the most important risk factor for developing head and neck cancers. Thus, in a smoker with $\mathrm{OL}$, it is necessary to differentiate between the so called stomatitis nicotina or "smoker's palate," and true leukoplakia. Stomatitis nicotina is a clinical picture characterized by whitish lesions on the surface of the soft palate or the floor of the mouth, in which tobacco smoke is the direct causal agent. These lesions have no malignant potential and result from the proliferative activity caused by tobacco smoke through the induction of epidermal growth factor receptor (EGFR), which subsequently activates cyclin D1. However, long-term exposure to this carcinogen may increase the frequency of mutations in the oral mucosa and, indirectly, cause genomic 
instability [5] which clinically manifests as loss of the fingerprint pattern and lack of resolution of the lesion after smoking cessation. In such cases the plaque could be considered as OL according to the above definition.

\section{Candida}

There is much debate about whether Candida species are implicated in the etiology or progression of OL. Different types of nitrosamine-producing Candida species (other than the common albicans variant) have been isolated from clinically non-homogeneous leukoplakias with histological dysplasia. The lesion does not resolve on eradication of this surface mycosis, but eliminating the fungus does lead to transformation of the high-risk non-homogeneous leukoplakia variant into a low-risk homogenous form. Fungal super infection is therefore considered as a significant risk factor for oncogenesis [6].

\section{Papillomavirus}

The possible implication of HPV in the etiology and potential for malignant transformation of oral precancerous lesions has been extensively studied. However, it remains open to debate whether the virus acts as an etiological agent in the development of leukoplakia or simply represents a super infection. The most studied HPV types, using in situ hybridization techniques, include types 6,11 , $16,18,31,33$, and 35 . It remains somewhat controversial however whether an association exists in the oral region. In a recent study, compared to the normal epithelium, a 2-to-3-fold higher incidence of HPV infection and 4-to-5 fold greater incidence of squamous cell carcinoma were detected in oral precancerous lesions. Highrisk HPV types are more frequently associated with squamous cell carcinoma than low-risk types; it can therefore be concluded that HPV-16 infection is a risk factor for malignant change in leukoplakia regardless of the harmful effects that tobacco smoke and alcohol might have on oral mucosa. The proliferative verrucous variant has often been associated with HPV co-infection, in particular with serotypes 16 and 18, given the oncogenic potential of these HPV types in other mucosa and the rapid progression of this clinical variant. However, using polymerase chain reaction (PCR) techniques [7] failed to detect HPV infection in a prospective study of 13 proliferative verrucous leukoplakias, placing in doubt the pathogenic relationship.

\section{Epstein-Barr virus}

Epstein-Barr virus has been implicated in a large number of cancers, and some studies have found it to be an etiologic factor in oral squamous cell carcinoma. In a recent preliminary study, no evidence was found of a causal link between proliferative verrucous leukoplakia and EBV; however, the study was conducted in a small number of patients, and so an etiologic association cannot be ruled out [8]. The development of OL as premalignant lesion involves different genetic events. This notion is supported by the fact that markers of genetic defects are differently expressed in different leukoplakias Activation of oncogenes and deletion and injuries to suppressor genes and genes responsible for DNA repair will all contribute to a defective functioning of the genome that governs cell division. Following a series of mutations, a malignant transformation may occur. For example, carcinogens such as tobacco may induce hyperkeratinisation, with the potential to revert following cessation, but at some stage, mutations will lead to an unrestrained proliferation and cell division.

\section{Clinical types of oral leukoplakia}

Two main clinical types of OL are recognized, being homogeneous and non homogeneous leukoplakia. The distinction of these is purely clinical, based on surface colour and morphological (thickness) characteristics, and do have some bearing on the outcome or prognosis. Homogeneous lesions are uniformly flat, thin and exhibit shallow cracks of the surface keratin. The risk of malignant transformation is relatively low. Non-homogeneous lesions carry a much higher risk of malignant transformation; Non homogeneous varieties include:

a) Speckled: mixed, white and red, but retaining predominantly white character

b) Nodular: small polypoid outgrowths, rounded red or white excrescences

c) Verrucous: wrinkled or corrugated surface appearance

d) Proliferative verrucous leukoplakia (PVL) presents with multiple, simultaneous leukoplakias; as the disease is visibly multifocal and frequently covers a wide area

\section{Histopathological features of oral leukoplakia}

In biopsies of OL a distinction can be made histopathologically between dysplastic and non-dysplastic lesions. The assessment and severity of dysplasia is based on architectural disturbance accompanied by cytological atypia. It should be emphasized that dysplasia is a spectrum and that no criteria exist to precisely divide this spectrum into mild, moderate and severe categories. Occasionally, a diagnosis of verrucous carcinoma, carcinoma in situ or invasive squamous cell carcinoma is made in the initial clinical presentation of leukoplakias; in such event the histopathological diagnosis replaces the clinical diagnosis of leukoplakia. It is well recognized that (proliferative) verrucous leukoplakia may show a spectrum of histopathological changes, ranging from hyperkeratosis with or without dysplasia to verrucous hyperplasia. The cliniopathological features that define dysplastic oral epithelium are listed below [4]:

a) Increased cell and nuclear volume and pleomorphism

b) Hyperplasia of basal cells

c) Presence of hyperchromatic nuclei

d) Prominent enlarged nucleoli

e) Increased nuclear: cytoplasmic ratio 
f) Premature keratinisation of independent cells

g) Increase in mitotic index

h) Mitosis above the basal layer

i) Loss of cell polarity

j) Decreased cell adhesion

k) Enlarged/fused epidermal ridges

\section{Diagnosis of oral leukoplakia}

The diagnosis of leukoplakia can be made at different levels of certainty, as a clinical term only or as a clinico-pathological diagnosis. A provisional diagnosis of leukoplakia is made when a predominantly white lesion at clinical examination cannot be clearly diagnosed as any other disease or disorder of the oral mucosa. A definitive diagnosis of leukoplakia is made when any aetiological cause, e.g. tobacco, Candida albicans, mechanical irritation, has been excluded and histopathologic examination has not disclosed any other specific disorder. At times, it may be difficult to exclude other disorders, even when a biopsy specimen is available. For instance, the histopathology of a white lesion may be compatible with both a clinical diagnosis of leukoplakia and lichen planus. In other words, some lesions are difficult to diagnose with certainty, even in the availability of a biopsy specimen. Nevertheless taking biopsy is strongly recommended not only to exclude any other specific disorder, but also to assess the absence or presence and degree of epithelial dysplasia or even carcinoma in situ or frank squamous cell carcinoma. The biopsy should be taken at the most clinically suspicious area, if any, such as redness, an area of surface thickening or a symptomatic area. Particularly in the case of a non-homogeneous leukoplakia an incisional biopsy may not be representative. In small leukoplakias, e.g. $<2-3 \mathrm{~cm}$, an excisional biopsy may be considered. The value of oral brush cytology is a subject of controversy, as is the use of toluidine blue, and other adjuncts. Histopathologic examination is at present still the gold standard for diagnostic purposes. The DNA ploidy measurements may be helpful in identifying lesions that carry a high risk of malignant transformation [9].

\section{Malignant transformation of oral leukoplakia}

An annual malignant transformation rate of leukoplakia of $0.3 \%$ has been reported in the Indian scenario [10]. Researchers from Western countries report somewhat higher figures of annual malignant transformation rate of approximately $1-2 \%$. It is well appreciated that this figure is much higher for non-homogeneous types, including proliferative verrucous leukoplakia. The latter entity probably nearly always transforms into one or more verrucous carcinomas or squamous cell carcinomas, but may do so in a protracted course of many years. Many leukoplakias probably may not progress over time; spontaneous regression is rare. Malignancies may develop at the site of treated or untreated leukoplakia, but may also occur elsewhere in the oral cavity or upper aerodigestive tract. The risk factors of for malignant transformation of OL are listed below [9]:

a) Female gender

b) Long duration of leukoplakia

c) Leukoplakia in non-smokers (idiopathic leukoplakia)

d) Location on the tongue and/or floor of the mouth

e) Size $>200 \mathrm{~mm}^{2}$

f) Non-homogeneous type

g) Presence of invasive Candida albicans

h) Presence of epithelial dysplasia

i) DNA aneuploidy

j) History of previous head and neck carcinoma

Of these risk factors, the presence of epithelial dysplasia often correlating with a clinically non-homogeneous erythroleukoplakic subtype are in general regarded the most important indicator of malignant potential. Nevertheless, it should be recognized that some dysplastic lesions may remain unchanged or may even show complete regression. Furthermore, carcinomatous transformation may also take place in non-dysplastic leukoplakia. In several studies from the Western world, the borders of the tongue and the floor of the mouth have been mentioned as high-risk sites, while in a study from Denmark also size was shown to be of importance, particularly when exceeding $200 \mathrm{~mm}$. In spite of tremendous progress in the field of molecular biology, there is as yet no single marker or set of markers that reliably enables to predict malignant transformation of leukoplakia in an individual patient with leukoplakia, perhaps with the exception of DNA ploidy measurements. The use of noninvasive genetic tests, using exfoliated or brushed cells of lesional tissue, or molecular markers from saliva may prove to be a step forward in the search for relevant prognostic markers [9].

\section{Management protocol of oral leukoplakia}

In the presence of possible aetiological factors, in particular a tobacco habit which may require professional habit intervention an observation period of not more than a somewhat arbitrarily chosen six weeks seems acceptable to observe a possible regression after elimination of such factors. Of course, it is well appreciated that complete regression may take much longer. On the other hand, one would not like to postpone a biopsy for too long.

Although there is no scientific evidence that treatment, of whatever type, truly prevents recurrences or the possible future development of a squamous cell carcinoma, it seems safe practice to actively treat leukoplakias, irrespective of the absence or presence of epithelial dysplasia, if feasible [9].

Treatment Guidelines for OL summarised by Longshore and Camisa [11] is as follows: 
a) Eliminate all contributing factors

b) Absence of dysplasia or presence of mild dysplasiasurgical excision/laser surgery of the lesions on the ventral/ lateral tongue, floor of the mouth, soft palate, and or pharynx. Close observation and follow-up for all other anatomic locations

c) Presence of moderate or severe dysplasia surgical excision or laser therapy is preferred treatments

d) Red lesions (erythroplakia or leukoerythroplakia) Surgical removal is best

e) Proliferative verrucous leukoplakia Complete surgical excision/laser surgery if possible

f) Follow-up for all lesions

\section{Conclusion}

A thorough knowledge on various aspects of oral potentially malignant disorders will help clinician to make appropriate diagnosis and plan treatment. Oral leukoplakia is mainly associated with tobacco usage either smoking or chewing. Thus, health education, counselling the individual and behavioural therapies are most successful approaches of prevention at a primary level.

\section{References}

1. Brouns ER, Baart JA, Bloemena E, Karagozoglu H, van der Waal I (2012) The relevance of uniform reporting in oral leukoplakia: Definition certainty factor and staging based on experience with 275 patients. Med Oral Patol Oral Cir Bucal 18(1): e19-e26.
2. Petti S (2003) Pooled estimate of world leukoplakia prevalence: a systematic review. Oral Oncol 39(8): 770-780.

3. Napier SS, Speight PM (2008) Natural history of potentially malignant oral lesions and conditions: an overview of the literature. J Oral Pathol Med 37: 1-10.

4. Martorell Calatayud A, Botella Estrada R, Bagán Sebastián JV, Sanmartín Jiménez O, Guillén Barona C (2009). Oral Leukoplakia: Clinical, Histopathologic, and Molecular Features and Therapeutic Approach. Actas Dermosifiliogr 100(8): 669-684.

5. Sudbø J and Reith A (2005) The evolution of predictive oncology and molecular-based therapy for oral cancer prevention. Int J Cancer 115(3): 339-345.

6. Reibel J (2003) Prognosis of oral pre-malignant lesions: significance of clinical, histopathological, and molecular biological characteristics. Crit Rev Oral Biol Med 14(1): 47-62.

7. Bagán JV, Jiménez Y, Murillo J, Gavaldá C, Poveda R, et al. (2007) Lack of association between proliferative verrucous leukoplakia and human papillomavirus infection. J Oral Maxillofac Surg 65(1): 46-49.

8. Bagán, JV, Jiménez Y, Murillo J, Poveda R, Díaz JM, et al. (2008) EpsteinBarr virus in oral proliferative verrucous leukoplakia and squamous cell carcinoma: A preliminary study. Med Oral Patol Oral Cir Bucal 13(2): E110-E113.

9. Diz P, Gorsky M, Johnson NW (2014) Oral leukoplakia and erythroplakia: a protocol for diagnosis and management. EAOM-Diagnostic and therapeutic protocols 1: 1-8.

10. Tanwar R, Dave A, Kalra M, Saluja P (2015) Non-Surgical Management of Oral Leukoplakia in Indian Scenario. University J Dent Scie 1(2): 49-54.

11. Longshore SJ, Camisa C (2003) Detection and management of premalignant oral leukoplakia. Dermatol Ther 15(3): 229-235.
(C) (i) This work is licensed under Creative

To Submit Your Article Click Here:

Submit Article
DOI: 10.32474/MADOHC.2018.01.000124

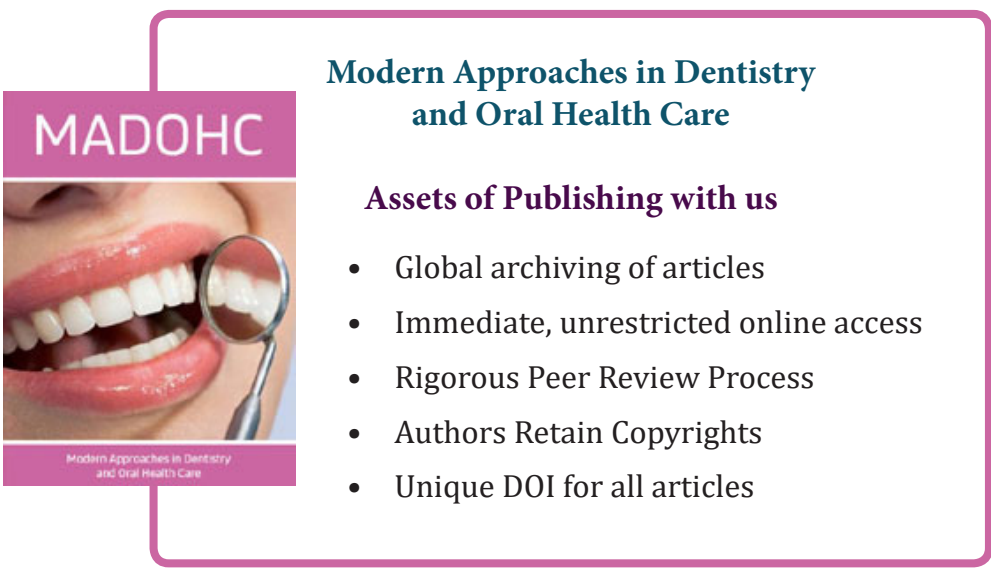

